\title{
Source monitoring in eyewitness memory: Implicit associations, suggestions, and episodic traces
}

\author{
STEVE T. HEKKANEN \\ University of Tampa, Tampa, Florida \\ and \\ CATHY MCEVOY \\ University of South Florida, Tampa, Florida
}

\begin{abstract}
Both the distinctiveness heuristic and discrepancy detection hypotheses were investigated by independently manipulating both schema consistency and incidental suggestion in an eyewitness memory paradigm. A sequence of slides was shown, followed by a postevent questionnaire that contained both schema-typical and schema-atypical information. Fifteen minutes later, a source-monitoring task was administered. In Experiment 1, the proportion of source misattribution errors was greater for schematypical items than for schema-atypical items, and the proportion of errors on suggested items was greater than that on control items. Suggestion affected schema-typical and schema-atypical items equally, providing no support for the predictions of either hypothesis. In Experiment 2, the interval between the questionnaire and the source test was manipulated. The results of Experiment 1 were replicated under the short delay, whereas the proportion of errors increased under the long delay. An associative network model involving two types of episodic traces was used to account for the results.
\end{abstract}

In the past decade, theoretical attention has focused on explaining various types of errors that occur during the recollection of an event. Source misattribution errors occur when the times, locations, or sources of items are improperly identified. The rate at which misattribution errors arise appears to be influenced by the use of the distinctiveness heuristic (Dodson \& Schacter, 2002; Schacter, Israel, \& Racine, 1999): a metacognitive strategy that helps determine which recollected items were part of the experience. Vivid details and distinctive information are assumed to be part of the memorial representation of an experience. When an item is recollected but lacks this information, it is identified as new or novel. Sometimes problems occur, and an item that is novel is identified as part of the experience, resulting in a recall, recognition, or source misattribution error. Presumably, the novel item was somehow encoded along with vivid details that made it indistinguishable from the items that were actually encoded from the experience. The purpose of the present study was to investigate the use of the distinctiveness heuristic in the interpretation of source misattribution errors in the context of an eyewitness paradigm.

Both Israel and Schacter (1997) and Schacter et al. (1999) evaluated the distinctiveness heuristic hypothesis

This research was supported by a Delo grant from the University of Tampa to S.T.H. and Grant AG13973 from the National Institute on Aging to C.M. We thank James Woodson and the reviewers for their helpful and insightful comments. Correspondence concerning this article should be sent to S. Hekkanen, Department of Psychology, 401 West Kennedy Blvd., University of Tampa, Tampa, FL 33606 (e-mail: shekkanen@ut.edu). using the Deese/Roediger-McDermott (DRM) paradigm. Roediger and McDermott's (1995) study exemplifies how this paradigm has been employed. They presented to participants several lists of words in succession. On a typical list (e.g., KID, ADULT, ADOLESCENT, etc.), each word was associatively related to a critical nonpresented word (e.g., CHILD). Even though the critical words were never presented during the study phase, the participants frequently identified them as having been presented.

The identification of those critical unstudied words as "old" items on a recognition test was assumed to be due to the activation of preexisting associates by the list words, making available implicit associative responses during the study phase (Roediger \& McDermott, 1995; Underwood, 1965). Because each of the list words was related to the critical word, that word was repeatedly activated during study. McEvoy, Nelson, and Komatsu (1999) provided evidence in support of this interpretation when they showed that the probability of falsely recognizing a critical word significantly increased as the strength of the connections between the list words and the critical word increased.

Two factors may be responsible for the false recognitions that are observed in a DRM paradigm: Implicit associative responses are activated by the list words during the study phase, and the use of the distinctiveness heuristic by the participants during the test phase would make it difficult for them to distinguish between studied words and critical words. To evaluate this possibility, Schacter et al. (1999) presented each word in the list with a descriptive picture and found that this technique resulted in a reduced number of critical words being falsely remem- 
bered as studied words. Presumably, the words from the lists were encoded with vivid pictorial details, whereas the critical words were not. During the memory test, these vivid details were used to distinguish between the studied words and the critical words, leading to improved accuracy.

The occurrence of implicit associative responses and the use of the distinctiveness heuristic may help to explain why participants in an eyewitness paradigm tend to include suggestions in their recollection of an experience. In a typical eyewitness testimony procedure (see, e.g., Zaragoza \& Lane, 1994), participants study a pictorial experience and then read a set of statements/questions with embedded misinformation that is always consistent with the pictorial experience. After a short delay, a sourcemonitoring task shows that the suggested misinformation is frequently identified as having occurred in the witnessed event.

The pictorial experience in an eyewitness paradigm is usually organized around a schema. For example, in Zaragoza and Lane's (1994) study, a workman comes to an office and is instructed to fix a chair by an assistant. When the assistant leaves, the workman rummages through the drawers and bags in the office and takes a calculator and money. At least two sources of memory errors could have occurred in this study. The first could have occurred when the pictorial experience was presented to the participants. The office schema and the items in the office activated semantically associated information in an associative network (Anderson, 1976, 1985), much as the words in the study phase of a DRM paradigm activate implicit associative responses (Roediger \& McDermott, 1995). Because the suggested items were consistent with the office schema, some of them may have been among the set of activated implicit items. The activation of implicit items while the pictorial sequence was being processed would have increased their levels of vividness. At the time of the test, the participants would have found the suggested typical items to be indistinguishable from the other items of the experience, because both the pictorial and the suggested typical items were encoded with distinctive details. Thus, some source misattributions in the Zaragoza and Lane study may have been the result of activating items during the processing of the experience and encoding those items with vivid details.

An additional source of memory errors in the Zaragoza and Lane (1994) study may have occurred when the questionnaire was presented to the participants. The script of the questionnaire paralleled the script of the experience except when misinformation was introduced. Because the participants were unaware that some of the items in the questionnaire contained misinformation, they would have been susceptible to encoding the misinformation, thus creating a suggestion effect. In addition, each of the suggested typical items could have activated items from the original experience, reinstating the memory of the experience and increasing the chance that suggested typical items would be encoded with vivid details. Thus, the source misattributions in Zaragoza and Lane's study and in other eyewitness studies may have been due both to the activation of implicit associative items during the encoding of the experience and to the combined influence of suggestion and activation that occurred during the reading of the questionnaire.

Smith and Studebaker's (1996) results provide some support for this interpretation. Participants in the first of their three experiments listened to an audiotaped description of an experience, followed by a questionnaire that contained information about details of the experience. The consistency of the subsequent information with the audio experience was manipulated in the following manner: The information biased the participants toward a typical interpretation of an experiential detail, biased them toward an atypical interpretation, or did not bias them toward any interpretation. After completing the questionnaire, the participants took a forced-choice recognition test in which each question had three alternative responses: a typical response, an atypical response, and a response that indicated that no such information was given in the audiotaped experience. The results showed an interaction between consistency and type of response: More typical responses were chosen after biasing by typical information, but when participants were biased by atypical information the proportion of typical responses decreased and the proportion of atypical responses increased. Smith and Studebaker concluded that prior knowledge predisposed the participants toward typical information.

Unfortunately, as the authors noted, the testing format may have caused the participants to pick typical rather than atypical responses, a problem that has been extensively examined by others (e.g., McCloskey \& Zaragoza, 1985). Even though the problem was investigated in their second and third experiments, the contributions of consistency and suggestion remain unclear. In the present set of experiments, we manipulated both degree of schema consistency and type of incidental suggestion in an eyewitness study, for two purposes: to evaluate the relative effects of the two factors and to determine whether they interact. The misinformation items in the experiment were either consistent with the pictorial experience (typical) or inconsistent with it (atypical); moreover, they either were suggested in the statements/questionnaire or served as control items. The participants' recollections of the experience were evaluated with a source-monitoring task.

As the participants view the pictorial experience in the present study, implicit associative items may be activated. Distinctive information may be encoded with those typical items that are not actually seen but are activated as implicit associative items. Thus, according to the distinctiveness heuristic hypothesis, more source misattributions should occur in the typical conditions than in the atypical conditions, and the manipulation of both incidental suggestion and schema consistency should produce an interaction between these two factors. The suggested typical items should reinstate the memory of the pictorial experience, allowing distinctive information to become encoded with these items. In contrast, the suggested atyp- 
ical items would not reinstate the experience because they are inconsistent with it. Thus, the suggested typical items, unlike the suggested atypical items, should be encoded with distinctive details, producing more source misattribution errors in the suggested typical condition than in the suggested atypical condition. At the time of test, there is the possibility that the control typical items could reactivate the memory of the experience, whereas the control atypical items would have no such possibility. If reactivation does occur, using a distinctive heuristic at the time of test may not be sufficient to distinguish among suggested typical items, witnessed items, and control typical items. Thus, some source misattribution errors should occur in the control typical condition, whereas virtually none should occur in the control atypical condition.

The discrepancy detection hypothesis is also relevant here (Tousignant, Hall, \& Loftus, 1986) because its predictions are similar to those derived from the distinctiveness heuristic hypothesis. According to the discrepancy detection hypothesis, the awareness of discrepancies between the pictorial experience and the postevent questionnaire minimizes the influence of misinformation, and as the degree of discrepancy increases so does the potential for rejecting the misinformation. Participants in the present study should reject atypical items more often than typical items. The appearance of atypical and typical items as suggestions on the postevent questionnaire should increase the acceptance of typical items and moderate the rejection of atypical items because the structure of the questionnaire parallels the experience, making both types of items plausible to varying degrees. When the atypical and typical items appear as control items on the test, the control atypical items should be easily rejected. However, the consistency between the control typical items and the experience should slightly moderate the rejection of these items. Thus, the predicted interaction between incidental suggestion and schema consistency would be similar to the interaction predicted from a distinctiveness heuristic perspective.

The two hypotheses are similar in that they both predict that atypical items should be rejected more often than typical items. However, the two hypotheses differ on when the rejection occurs. The distinctiveness heuristic hypothesis states that rejection occurs while the participants are responding to the test stimuli. According to this hypothesis, the amount of distinctive information encoded with each test item varies: Typical items are encoded with significant amounts of distinctive details from the experience, and atypical items are encoded with no distinctive information from the experience. At the time of test, items with distinctive details would be accepted as part of the experience and items without distinctive details would be rejected. Thus, the atypical items would be rejected and the process of rejecting these items would occur at the time of test. In contrast, the discrepancy detection hypothesis states that rejection occurs primarily during encoding of the information. For example, as a participant encounters misinformation on the postevent questionnaire, rejection of the misinformation should occur at varying rates and the highest rejection rate should occur for the atypical items. Thus, this perspective maintains that the rejection of atypical items starts before the test is taken.

\section{EXPERIMENT 1}

\section{Method}

Participants. Undergraduate students were recruited and given extra course credit for their participation. Groups of 2-15 students were tested in both morning and afternoon sessions. There was a total of 120 participants.

Design. The primary design for this experiment was a $2 \times 2$ within-subjects factorial. Schema consistency (schema typical vs. schema atypical) was crossed with incidental suggestion (suggested vs. control). The items for these four conditions were from the nonslide critical set and never appeared in the slide presentation. The items for the suggested conditions were mentioned in the postevent questionnaire that immediately followed the slide presentation. The items for the control conditions never appeared in the postevent questionnaire. Both the typical and atypical items were counterbalanced across the suggested and control conditions using different groups of participants.

Items from the slide critical set were names of objects that were part of the slide presentation. For each participant, these items appeared in one of three slide critical set conditions: slide (in which the participants viewed the items on slides), read (in which the participants read the names of the items), and neither (in which the items were not presented to the participants). When slide items were not in the slide condition, they were either in the read condition or in the neither condition. These items were counterbalanced across the three conditions through the use of different groups of participants.

There were four possible responses on the source-monitoring test. The participant could respond "saw only," "read only," "both," or "neither." Choosing "saw only" indicated that the participant felt the test item had appeared only in the slide show; choosing "read only" indicated that the participant felt the item had appeared only in the questionnaire. The "both" response indicated that the participant thought the item had appeared in both the slide presentation and the postevent questionnaire. The "neither" response was selected when the participant thought that the item had not appeared in either the slides or the questionnaire.

Materials. The schema-typical and schema-atypical items were obtained from norms that were collected by the authors. Eighty items were chosen that represented objects that, in the authors' judgment, varied in their likelihood of appearing in a typical office setting. About one third definitely would be found in an office, and about one third definitely would not be found. The remaining items fell between the two extremes. The participants in the norming study were told to think about a typical office setting in which people are busily working and to indicate whether each item is likely or unlikely to be found in an office, on a five-point Likert scale. A rating of 1 indicated very likely, and a rating of 5 indicated very unlikely. The 80 items were randomly divided among three sheets, and each sheet had two random arrangements. The ordering of the sheets was also randomized. Data were collected from 87 participants. The 12 schema-typical items had a mean typicality rating of $1.51(S D=0.34)$; the 12 schema-atypical items had a mean typicality rating of $4.23(S D=0.39)$. These items can be found in the Appendix.

The 79 slides were obtained from the Zaragoza and Lane (1994) study and depicted a workman entering a room, repairing a chair, and then stealing an envelope full of money and a calculator. Zaragoza 
and Lane used 6 of the 79 slides as critical slide items. In the present study, that number was increased to 9 after careful examination of all the slides. Each of the 9 slides identified as critical contained one of nine critical items that appeared in no other slide in the sequence. Of the nine critical items in the slide critical set, three actually appeared in the slide sequence, three were suggested items, and three served as "neither" items for any given participant. When an item appeared in either the suggested or the neither condition, the slide in which that item appeared was either removed from the viewing sequence or replaced with an alternate slide that did not contain the critical item. The nine critical slide items were counterbalanced across the three conditions using different groups of participants. These items were used to calculate the recognition accuracy of veridical memories.

The 12 schema-typical and 12 schema-atypical items from the nonslide critical set served only in the suggested and control conditions and never appeared in the slide sequence. Half of the schematypical and half of the schema-atypical items served in the suggested conditions, and the remaining items served in the control conditions. The schema-typical items and the schema-atypical items were counterbalanced across the suggested and control conditions by varying the wording in the postevent questions, and hence they were counterbalanced within each group of participants. The typical and atypical items were used to calculate the source misattribution rates.

The questionnaire contained 16 sets of statements, each ending with a question. Each question was preceded by one, two, or three statements. The statements described objects and sequences of actions, and the questions required one-word or two-word responses. For example, the following was a typical set:

As the man carried the chair back to its place behind the desk, he caught sight of a shopping bag with balloons attached to it on the floor near the desk. He proceeded to take various articles out of the bag, including a blue sweater and a Wilson tennis racket. He placed them near the aspirin bottle on the desk. How many items did he remove?

The questionnaire was used to introduce the suggested items. Fifteen of the 16 sets of statements were used to distribute the 15 suggestions: three items from the slide critical set, six schema-typical items from the nonslide critical set, and six schema-atypical items from the nonslide critical set. The last set of statements contained no suggested items. None of the suggested items in the nonslide critical set referred to objects that appeared in the slides. In addition, the schema-typical and schema-atypical nonslide critical items appeared only in the statements preceding the question.

The source memory test consisted of all critical items -9 from the slide critical set and 24 nonslide critical items that were schema typical or schema atypical - and 13 filler items, for a total of 46 items. The sequence of the items was randomized. The test was presented auditorily, and the participants marked on a response sheet whether each item was "saw only," "read only," "both," or "neither."

Procedure. The general procedure and instructions that were used in Zaragoza and Lane (1994) were also employed in this experiment. The participants were informed that the experiment had to do with the interpretation of complex events and that their task was to view the sequence of slides and to try to determine the incident portrayed in the slides. They were also told that the slides would be presented at a 4-sec rate and to be careful not to look away during the presentation.

After the 5.5-min slide presentation, the participants received the postevent questionnaire and were instructed to answer each of the questions even if they had to guess. On completing the questions, they immediately progressed to the filler task. They were allowed 15 min to complete the forms.

The instructions for the final source-monitoring test that were used in Hekkanen and McEvoy (2002) were also used in this study. They closely paralleled the instructions from Zaragoza and Lane
(1994) with one exception: The descriptors for the responses were "read only," "saw only," "both," and "neither." The instructions were provided in written form and read aloud to the participants. After the questions were answered, the participants completed the 46item source-monitoring test.

\section{Results}

The proportions of "saw only," "read only," "both," and "neither" responses that were made to the various test items are shown in Table 1. These data indicate that 35\% of the slide items were correctly classified as seen, $54 \%$ of the read items were correctly classified as read, and $69 \%$ of the "neither" items were correctly identified as neither seen nor read. The mean response rates for the nonslide critical set conditions are also shown in Table 1.

Our definition of a source misattribution error was consistent with what is typically used in eyewitness studies. For example, Zaragoza and Lane (1994) defined a misattribution error as an item on the source-monitoring test that was identified as either seen only or both seen and read, when it had in fact only been read. Our results showed that $28 \%$ of the schema-typical suggested items and $20 \%$ of the schema-typical control items elicited source misattribution errors. In addition, $13 \%$ of the schema-atypical suggestions and $4 \%$ of the schemaatypical control items also elicited errors.

For all statistical tests, a significance level of $p=.05$ was adopted. The proportions of source misattribution errors for the nonslide critical items were subjected to a two-way (schema-typical vs. schema-atypical $\times$ suggested vs. control) repeated measures analysis of variance (ANOVA). The main effect of incidental suggestion was significant $\left[F(1,119)=24.01, M S_{\mathrm{e}}=0.03\right]$, as was the main effect of schema consistency $[F(1,119)=100.38$, $\left.M S_{\mathrm{e}}=0.03\right]$, but the interaction between the two factors was not significant $\left[F(1,119)=0.01, M S_{\mathrm{e}}=0.02\right]$. The participants committed more source misattribution errors on the suggested items $(M=.20, S D=.24)$ than on the control items $(M=.12, S D=.19)$. In addition, more misattribution errors occurred when the control and suggested items were schema typical $(M=.24, S D=.24)$ than when they were schema atypical $(M=.08, S D=.16)$.

Table 1

Experiment 1: Mean Proportions of Responses to Slide Critical Set Items and Nonslide Critical Set Items

\begin{tabular}{lcccc}
\hline \multicolumn{1}{c}{ Condition } & \multicolumn{4}{c}{ Response Type } \\
\cline { 2 - 5 } & Saw Only & Read Only & Both & Neither \\
\hline Slide critical set & & & & \\
$\quad$ Slide & .35 & .07 & .17 & .41 \\
Read & .04 & .54 & .29 & .13 \\
$\quad$ Neither & .12 & .10 & .09 & .69 \\
Nonslide critical set & & & & \\
$\quad$ Schema-typical suggested & .15 & .31 & .13 & .41 \\
Schema-typical control & .18 & .03 & .02 & .77 \\
Schema-atypical suggested & .03 & .55 & .10 & .32 \\
Schema-atypical control & .03 & .04 & .01 & .92 \\
\hline
\end{tabular}


Incidental suggestion. The size of the suggestion effect was calculated in two ways. The mean misattribution rate of the control typical condition was subtracted from that of the suggested typical condition, providing a mean difference of .08. In addition, the mean misattribution rate of the control atypical condition was subtracted from that of the suggested atypical condition, providing a mean difference of .09 . That the two estimates were almost identical in size was surprising because different conditions were used for each estimate. The first estimate was based on responses to items from the typical conditions, whereas the second estimate was based on responses to items from the atypical conditions. Given that the items were different and that counterbalancing could not be used between the typical and the atypical conditions, the odds were stacked against arriving at approximately the same value.

Schema consistency. The size of the consistency effect was also evaluated using two mean difference scores. The first estimate involved subtracting the mean misattribution rate of the suggested atypical condition from that of the suggested typical condition; the mean difference was .15. The second estimate was obtained by subtracting the mean misattribution rate of the control atypical condition from that of the control typical condition; the mean difference was .16. Again, the convergence of the two estimates was somewhat surprising. Even though the results from one typical and one atypical condition were used in each estimate, one estimate was derived from the responses to suggested items whereas the other was derived from responses to control items.

Analysis for veridical memories. Correct identifications of the slide and read items were compared using a paired $t$-test analysis, and a significant difference was found $[t(119)=-4.99]$. Accuracy was greater for read items $(M=.54, S D=.34)$ than for slide items $(M=.35$, $S D=.26)$.

\section{Discussion}

Even though the results of Experiment 1 were straightforward, some of them were unexpected. Typical items produced more source misattribution errors than did atypical items, confirming the prediction of both the distinctiveness heuristic and discrepancy detection hypotheses about the main effect of schema consistency. In addition, more errors occurred when items were suggested than when they were not suggested (control items), supporting the presence of a suggestion effect. However, contrary to the predictions of both hypothesis, schema consistency and incidental suggestion did not interact with one another. We were surprised to find a lack of interaction and almost identical rates of source misattributions both for suggested items regardless of schema typicality and for schema-typical items regardless of suggestion.

The proportion of source misattributions that was attributable to schema consistency was approximately 16 . Schema consistency led to source errors, and the effect did not depend on the items' actually being suggested.
When an item was schema typical, the chance of its being falsely recognized as part of the pictorial experience increased because the participants relied on schema consistency during the source-monitoring test, or because these items had been activated during the pictorial experience. These results are consistent with what has been found in past research concerning schemas and schematypical information (see, e.g., Belli, 1988; Bower, Black, \& Turner, 1979; Brewer \& Treyens, 1981).

The effect of suggestion on the source misattribution rate was calculated by finding the mean difference between suggested and control conditions, and the estimate was approximately .09. Because incidental suggestion did not interact with schema consistency, the effect of suggestion affected schema-typical items and schemaatypical items equally. For instance, scissors, a schematypical item, was no easier to suggest than DOLLS, a schema-atypical item. Suggesting the presence of an object increases its likelihood of being recollected as part of a witnessed event, regardless of the object's semantic consistency with the witnessed event. The present research, unlike previous research, has shown that incidental suggestion and schema consistency can produce distinct effects and that they do not necessarily interact with each other, at least in an eyewitness study.

The lack of an interaction between schema consistency and incidental suggestion in the present study challenges the predictions derived from our formulation of both the distinctiveness heuristic hypothesis and the discrepancy detection hypothesis. We expected that when the participants in the present experiment read the questionnaire with the schema-typical items, the typical items would reinstate the original experience and increase the vivid details encoded with them. We also expected that when the participants used the distinctiveness heuristic at test, they would find it difficult to distinguish between witnessed information and schema-typical items but easy to distinguish between witnessed information and schema-atypical items, and that this effect would be intensified for the suggested items. The results showed that this did not happen, suggesting that the participants were not using the distinctiveness heuristic to rule out suggested items any more than they used it to rule out control items.

From a discrepancy detection perspective, schemaatypical information should have been rejected more often than schema-typical information, particularly for suggested items, but this was not the case. The results showed that the rejection rates were the same for the two types of items; therefore, discrepancy detection did not differentially reduce source misattribution errors on suggested items in the present experiment. Even though the results of the present study did not support either hypothesis, the use of these strategies by the participants has been shown in other experiments (Schacter et al., 1999; Tousignant et al., 1986), so future research needs to elucidate the conditions under which each of these strategies does and does not occur. 
From other theoretical perspectives (Friedman, 1979; Schank, 1982), awareness of a discrepancy causes additional processing rather than rejection. For example, Schank proposed that participants who are using a schema to encode items would take longer to process atypical items than typical items because differences between the items and the schema must be reconciled. In the present experiment, the items in the suggested atypical condition were inconsistent with the pictorial schema of the witnessed event, so the participants should have taken longer to process them than to process typical items. This additional processing would have led to the schema-atypical items' being remembered differently from the schematypical items. Because the results showed no interaction between schema consistency and incidental suggestion, both types of items received comparable processing.

One possible explanation for the results of the present experiment involves the theoretical consideration of two types of memory traces. The effect of consistency that was observed in the present experiment suggests that semantic activation was occurring in an associative network. The typical items were semantically related to the schema of the witnessed event, and the semantic relations between these items and the schema were responsible for the large proportion of source misattributions. As the participants were watching the slide sequence, the items and the general schema of the experience activated related items within their associative networks (see, e.g., Anderson, 1976, 1985). Networks have both generic and episodic nodes, and the nodes are associatively linked to each other. The generic nodes include categories, schemas, and other propositional knowledge, whereas the episodic nodes represent specific instances. Activation of an item in a schema can spread to the other items in the schema and to other related schemas and knowledge. The spread of activation that was initiated by the witnessed event of the present study activated semantically related information, such as implicit associative items, and some of these items were related to the typical items that appeared on the source-monitoring test.

The suggestion effect in the present experiment, in contrast with the consistency effect, was more episodic. The results showed that it did not matter whether the suggested items were typical or atypical of the schema for the witnessed event. The sizes of the suggestion effects were almost identical. Because the semantic relations between the suggested items and the witnessed experience did not influence the results, this effect apparently is not knowledge based. Instead, the information from the questionnaire must have been encoded into an episodic trace in an associative network (see, e.g., Anderson, 1976, 1985), perhaps similar to a "weird list" (Friedman, 1979; Schank, 1982). According to frame theory, information that is inconsistent with the content structure of a frame is placed on an attached list of unexpected information. The results of the present study appear to support the view that the memories of the suggestions are distinct from the semantic portion of the associative network, be- cause there was no effect of schema activation on the encoding of suggested items: Suggested typical items apparently did not reinstate the witnessed event, and the schema of the event did not differentially influence the encoding of the suggested items.

The suggestion effect might be similar to the forced confabulation effect observed by Zaragoza, Payment, Ackil, Drivdahl, and Beck (2001). When participants in their study were required to consider blatantly false events, the researchers found that a significant number of such events were accepted as "real" experiences. Similarities between the procedures of their study and the procedures of the present study may support the possibility that the two effects are related. In the present experiment, the participants were asked to read the questionnaire and answer all questions even if they had to guess, whereas in the Zaragoza et al. study, the participants viewed a movie clip and then were required to answer questions about false events even if they had to guess. In their study, the participants misremembered witnessing the false events, and the size of the effect increased with the presence of confirmatory feedback. Even though the participants in the present experiment were never questioned about the misinformation as were the participants in the Zaragoza et al. study, the act of responding to the questions may have been perceived as confirmatory feedback, influencing the encoding of the information from the questions and the misinformation from the statements that preceded each question.

\section{EXPERIMENT 2}

The second experiment was similar to the first except that all participants were tested twice: $15 \mathrm{~min}$ after the postevent misinformation task and again $48 \mathrm{~h}$ later. The 15-min delay condition was included in Experiment 2 in an attempt to replicate Experiment 1, and the longer delay provided a means of evaluating additional hypotheses.

The first hypothesis concerned the lack of an interaction between schema consistency and incidental suggestion in Experiment 1. Testing the participants in Experiment 1 shortly after the postevent questionnaire may not have provided sufficient time for a schema consistency and incidental suggestion interaction to develop. Our original expectation was that the suggested typical items would reinstate the witnessed experience and the suggested atypical items would not; however, the results of Experiment 1 did not support this expectation. Even though the suggested typical items did not appear to reinstate the witnessed experience, there is the possibility that given adequate time they may eventually have integrated with the schema of the witnessed event. When the delay between the questionnaire and the source-monitoring test was increased in Experiment 2, we expected that suggested typical information would integrate into the schema from the witnessed event but suggested atypical information would not, increasing source misattribution errors for suggested typical items more than for suggested 
atypical items. Thus, an interaction among delay, schema consistency, and incidental suggestion would be expected.

The second hypothesis was related to the schema consistency and delay interaction. According to an associative network perspective, as the interval between the witnessed event and the source-monitoring task increases, the importance of schemas to the recollection of the event should increase. However, the results of previous research are unclear on this matter. Both Thapar and McDermott (2001) and Lampinen and Schwartz (2000) showed that both false memories and veridical memories decline over time. However, Lampinen, Copeland, and Neuschatz (2001) used two different delays along with an incidental learning procedure. They found that veridical recognition of typical items stayed the same, whereas false memory rates for both typical and atypical objects increased, with the greatest increase occurring for typical objects. Tuckey and Brewer (2003) used an eyewitnesstype procedure, and when they analyzed "remember" judgments they found that the number of incorrect responses increased when the first interview was delayed 4 weeks but there was no difference for schema-typical and schema-atypical items. In the present experiment, the schema that was derived from the slide experience was expected to influence performance on the sourcemonitoring task and, after a long delay, reliance on the schema would increase. The second hypothesis was that the increase in source misattribution errors after a long delay would be greater in the typical conditions than in the atypical conditions.

Finally, we investigated a third possibility: Episodic traces may persist, as the results of some studies have shown. For example, Salasoo, Shiffrin, and Feustel (1985) showed in a word recognition study that the identification of a nonword is enhanced by prior experience with the stimulus and that this effect can last a year. Using an eyewitness testimony procedure, Higham (1998) compared the results of two studies and showed that the suggestion effect increased after a long delay. Based on these results, a third hypothesis was that the suggestion effect would increase after a 48-h delay.

\section{Method}

Participants. Students in general psychology classes served as participants in the second experiment. None of them had served in Experiment 1 . They were tested during class time, and the 2-day testing format occurred on either a Monday/Wednesday schedule or a Tuesday/Thursday schedule. A total of 114 students participated in the experiment.

Design and Materials. The primary design for this experiment was identical to that of Experiment 1, except for the following details. The design was a $2 \times 2 \times 2$ within-subjects factorial in which schema consistency (schema typical vs. schema atypical) was crossed with both incidental suggestion (suggested vs. control) and delay (15 min vs. $48 \mathrm{~h}$ ), for a total of eight conditions rather than four. In addition, the participants were tested $15 \mathrm{~min}$ after the postevent questionnaire and again $48 \mathrm{~h}$ later. The first delay was identical to that used in Experiment 1.

The materials were identical to those of Experiment 1, with two exceptions. First, the participants of Experiment 1 filled out forms during the 15-min delay between the postevent questionnaire and testing. In Experiment 2, the 15-min period was filled with lecture material. The second exception concerned the randomization of the items for the two testing periods. In each test, a different random presentation of the item list was used.

Procedure. The same general procedure, counterbalancing techniques, and instructions used in Experiment 1 were employed here. The experimenters always arrived at the beginning of each class, instructed the participants, presented the slides, and had the participants fill out the postevent questionnaire. They thanked the participants for their help and left the classroom for $15 \mathrm{~min}$. The professor had been previously instructed to lecture to the students for $15 \mathrm{~min}$. At the end of the delay, the experimenters reentered the classroom and administered the source-monitoring test. After test-

Table 2

Experiment 2: Mean Proportions of Responses to Slide Critical Set Items and Nonslide Critical Set Items for the Two Delays

\begin{tabular}{llccc}
\hline \multicolumn{1}{c}{ Condition } & \multicolumn{4}{c}{ Response Type } \\
\cline { 2 - 5 } & Saw Only & Read Only & Both & Neither \\
\hline Slide critical set, 15-min delay & & & & \\
$\quad$ Slide & .35 & .04 & .21 & .40 \\
Read & .04 & .49 & .33 & .14 \\
Neither & .10 & .08 & .12 & .70 \\
Slide critical set, 48-h delay & & & & \\
Slide & .23 & .14 & .34 & .29 \\
Read & .06 & .40 & .41 & .13 \\
Neither & .12 & .23 & .23 & .42 \\
Nonslide critical set, 15-min delay & & & & \\
Schema-typical suggested & .18 & .29 & .16 & .37 \\
Schema-typical control & .21 & .04 & .04 & .71 \\
Schema-atypical suggested & .05 & .48 & .11 & .36 \\
Schema-atypical control & .06 & .03 & .01 & .90 \\
Nonslide critical set, 48-h delay & & & & \\
$\quad$ Schema-typical suggested & .18 & .24 & .20 & .38 \\
Schema-typical control & .18 & .18 & .11 & .53 \\
$\quad$ Schema-atypical suggested & .05 & .43 & .11 & .41 \\
Schema-atypical control & .05 & .11 & .04 & .80 \\
\hline
\end{tabular}


ing, the students were thanked for their participation and told that the experimenters were interested in how well they could remember details.

The experimenters returned to each classroom $48 \mathrm{~h}$ later. The participants were given the same testing instructions and a different randomization of the source-memory item list. They were thanked for their participation and told that their ability to recall details at the two different delays would be compared.

\section{Results}

The proportions of "saw only," "read only," "both," and "neither" responses that were made to the various test items at each delay are shown in Table 2. The data indicated that $35 \%$ of the slide items were correctly classified as seen and $49 \%$ of the read items were correctly classified as read after the 15-min delay. The percentages changed to $23 \%$ and $40 \%$, respectively, after a 48 -h delay. The mean response rates for each nonslide critical set condition at the two delays are also shown in Table 2 .

The source misattribution error rates for the various conditions were calculated. The means at the short delay showed that $34 \%$ of the schema-typical suggested items and $25 \%$ of the schema-typical control items were source misattribution errors. In addition, $16 \%$ of the schemaatypical suggested items and $7 \%$ of the schema-atypical control items were also source errors. After $48 \mathrm{~h}$, the percentages were $38 \%, 29 \%, 16 \%$, and $9 \%$, respectively.

The proportions of source misattribution errors for the nonslide critical items were subjected to a $2 \times 2 \times 2$ repeated measures ANOVA. The within-subjects factors were schema consistency (schema typical vs. schema atypical), incidental suggestion (suggested vs. control), and delay (15 min vs. $48 \mathrm{~h}$ ). The main effect of incidental suggestion was significant $\left[F(1,113)=39.80, M S_{\mathrm{e}}=\right.$ $0.04]$; more suggested items $(M=.26, S D=.25)$ than control items $(M=.18, S D=.22)$ were falsely identified. The main effect of consistency was significant $[F(1,113)=$ 184.65, $\left.M S_{\mathrm{e}}=0.05\right]$; more typical items $(M=.31, S D=$ $.25)$ than atypical items $(M=.12, S D=.18)$ were falsely identified. The main effect of delay was also significant $\left[F(1,113)=5.90, M S_{\mathrm{e}}=0.03\right]$; more items were falsely identified as being part of the witnessed event after the long delay $(M=.23, S D=.24)$ than after the short delay $(M=.20, S D=.24)$. There was a marginally significant interaction between delay and schema consistency $\left[F(1,113)=3.79, M S_{\mathrm{e}}=0.01, p=.054\right]$. The rise in the proportion of falsely identified items tended to be greater for typical items than for atypical items. At the short delay, mean proportions were $.29(S D=.25)$ for the typical condition and $.11(S D=.18)$ for the atypical condition. At the long delay, the mean proportions were $.33(S D=.25)$ and $.13(S D=.18)$, respectively. No other sources of variance had significant effects.

Incidental suggestion. For the short delay, subtracting the mean misattribution rates for suggested typical and control typical conditions and subtracting the mean misattribution rates for the suggested atypical and control atypical conditions provided the same estimate of .09. At the long delay, the estimates were .09 and .07 , re- spectively. Thus, the effect of suggestion did not appear to change over time.

Schema consistency. The size of the consistency effect was also evaluated using two estimates. The first estimate involved subtracting the mean misattribution rate of the suggested atypical condition from that of the suggested typical condition, and the second estimate involved subtracting the mean misattribution rate of the control atypical condition from that of the control typical condition. The two estimates for the short delay were both .18, and for the long delay they were .22 and .20 , respectively.

Analysis of veridical memories. Source identification accuracy for the slide and read items was examined for the two delays. A $2 \times 2$ repeated measures ANOVA was used to evaluate the data. Both delay (short vs. long) and items (slides vs. read items) had two levels. There were only two significant effects: delay $[F(1,113)=$ $\left.32.48, M S_{\mathrm{e}}=0.04\right]$ and items $\left[F(1,113)=18.35 . M S_{\mathrm{e}}=\right.$ $0.15]$. Correct source identification accuracy was greater at the short delay $(M=.42, S D=.32)$ than at the long delay $(M=.32, S D=.31)$. The effect of items showed that the accuracy rate for read items $(M=.44, S D=.33)$ was greater than that for slide items $(M=.29, S D=.28)$.

\section{Discussion}

The results of Experiment 2 replicated and extended the results of Experiment 1 . There was no interaction between incidental suggestion and schema consistency or among delay, incidental suggestion, and schema consistency. Thus, there was no support for the first hypothesis that suggesting typical items would be easier than suggesting atypical items. The effects of suggestion and consistency appear, once again, to be distinct from each other. Typical items created more source misattribution errors than atypical items, and suggestion influenced typical and atypical items alike. The effect of suggestion did not vary with typicality within the constraints of the present experiment.

The second hypothesis concerned the delay and schema consistency interaction. The expectation was that the schema from the witnessed event would become more important over time: As the delay increased, the proportion of misattribution errors in the typical conditions would increase faster than that in the atypical conditions. The results showed a marginally significant interaction between delay and schema consistency, with the proportion of errors increasing in each condition over time but the rate of increase in the typical conditions appearing to be twice as great. Even though the interaction between delay and schema consistency only approached significance, the significant effect of delay showed that the proportion of source errors increased as delay increased. This increase appeared to be due to both the suggestion and consistency effects with, perhaps, a somewhat greater contribution coming from the typical items.

Both Lampinen et al. (2001) and Tuckey and Brewer (2003) found a larger increase in the proportion of errors as the interval between study and test increased than was 
observed in the present study. Several methodological differences between their studies and the present one preclude identifying which factors were responsible for the differences in results. The prior studies used remember/ know judgments whereas we used a source-monitoring task, and the intervals between study and test varied. A further difference is that all the participants in the present study were tested at both delays using different random orderings of the same test items, whereas in the other studies test delay was manipulated between participants. Perhaps participating in the first test inoculated the participants against making large numbers of source errors on the second test. That notwithstanding, the pattern of results from the present study was consistent with the general pattern in both Lampinen et al. and Tuckey and Brewer. Apparently, the number of false identifications increases as delay increases.

The third hypothesis of the present experiment concerned whether or not the suggestion effect would increase after $48 \mathrm{~h}$. The results showed that the effect of suggestion did not interact with the effect of delay. Incidental suggestion, which accounted for $7 \%-9 \%$ of the responses at each delay, had a small but persistent influence on the responding of the participants. The nature of this effect was probably episode-based because incidental suggestion failed to interact with schema consistency. The presence of an interaction would have suggested that relational information existed between the schema of the witnessed event and the episodic trace. Because there was no interaction, there must be some degree of independence between the schema and the episodic trace(s).

The results also showed that these episodic traces did not change over $48 \mathrm{~h}$. There are two plausible reasons for this lack of change. One possibility is that the traces were durable and a decline would be observed only if the delay were extended beyond $48 \mathrm{~h}$. Another possibility is that changes in the size of the suggestion effect were attenuated by testing the participants twice. Repetition of an item is known to produce durable episodic traces (Salasoo et al., 1985), and it is possible that the repetition of the test items (not that of the suggestions) led to durable traces in the present study - a finding that would have both theoretical and practical importance. This finding would be rather interesting because it implies that an item can be suggested once and, by repeated testing of the item without repeating the suggestion, the episodic trace can be maintained.

\section{GENERAL DISCUSSION}

The results of the present experiments extend the findings of previous eyewitness testimony research. The general results of past research have shown that suggested typical information is incorporated into the recollection of a witnessed event. The present results showed that when an eyewitness testimony procedure is used, two effects occur. There is an effect of consistency, in that items that are consistent with the witnessed event more readily create source misattribution errors than do items that are inconsistent. In addition, there is an effect of suggestion that is distinct from the effect of consistency. Items can be suggested to occur in a witnessed event, and there is some likelihood that they will be recollected as having occurred irrespective of whether the suggested items are consistent or inconsistent with the event. Given that our results showed that source misattributions did not decline with time whereas veridical memories underwent a significant reduction in accessibility, memories for misinformation, though similar in many ways to veridical memories, are not entirely the same.

These findings provide some insights into how associative networks, episodic traces, and source monitoring interact to influence eyewitness testimony. Associative networks contain various generic nodes that represent schemas, categories, and other semantic information, with associative links between the nodes that vary in strength. The strengths of the links influence the amount of activation accruing to one node from another. In addition, the network contains specific instances of generic nodes, which are referred to as episodic nodes. The source monitoring framework (Mitchell \& Johnson, 2000) suggests that these links become activated when encoding, retrieval, and decision processes are engaged. The accuracy of the source judgment varies with encoding strategies, the amount and types of details encoded, the accessibility and uniqueness of the details, decision strategies, and retrieval strategies (Lindsay, 1994; Mitchell \& Johnson, 2000). False memories occur either when the episodic details do not clearly distinguish the source of a memory or when the decision and retrieval strategies do not make use of the source details.

In the present eyewitness study, the witnessed event of a business office activated a business office generic schema. The event itself was encoded into an episodic trace linked to the generic office schema. As the trace was being encoded, the generic schema activated semantically related items. The activation of these items by the generic schema increased the likelihood that they would be introduced into the episodic trace of the business office. Some of these items were the typical items that occurred on the test, either as suggested items or as control items. When the participants performed the sourcemonitoring task, they identified typical items as seen because they had been encoded into the episodic trace.

An explanation of the suggestion effect requires the introduction of another theoretical structure, akin to the "weird list" in frame theory (Friedman, 1979; Schank, 1982). According to frame theory, an event that contains information that departs from its generic node would be stored in an attached "weird list." We are proposing that as the participants saw the slides, they developed an episodic trace from the pictorial experience. When they read the questionnaire, departures from this trace were acknowledged by encoding of the information into a secondary episodic trace, which we have termed the ancillary episodic trace. Information, such as that in the form of 
suggested typical and atypical items, would be stored in this trace because it was never part of the pictorial story.

Unlike the relation between the "weird list" and the generic schema in frame theory, we propose that the ancillary episodic trace is linked to the episodic trace of the event. In both frame theory and associative network models (see, e.g., Anderson, 1976, 1985), the generic schema controls what is and is not included in the episodic trace, so schema-atypical items in our experiment would have been excluded at a higher rate than schema-typical items. To the contrary, the results from our experiment showed that the two types of items were treated equally. In addition, the episodic trace and the ancillary episodic trace appear to be separable because, during the viewing of the slides, the generic schema activated implicit associative items that caused the misattribution of typical items as "seen," but the activation of these implicit items did not seem to differentially influence how the suggested items were processed by the participants when they read the questionnaire. We also propose that because the relation between the two episodic traces is strong, activating one trace results in activation of the other trace, which would make it difficult to distinguish between the contents of the two traces.

Even though the independent effects of consistency and suggestion pose an interesting challenge for the distinctiveness heuristic hypothesis, this finding does not suggest that our participants were not using this strategy. When the participants were encoding the information from the slides, the generic schema that was directing the processing of the pictorial information activated semantically related information, some of which included the suggested typical items. Both the witnessed information and the activated information were encoded into the same episodic trace. Even if the participants were using the distinctiveness heuristic, they would not have been able to distinguish between the typical items and the witnessed items because both were encoded into the same trace. In addition, when both typical and atypical items were suggested to the participants, they were encoded into the ancillary episodic trace. Given that the ancillary episodic trace was linked to the episodic trace, the participants may have found it impossible to distinguish between the two sets of items because the distinctive details for the two traces may not have been sufficiently different.

Incorporating the ancillary episodic trace into an associative network model can help to explain how various factors, such as extended processing, level of dissociation, use of visual imagery, and forced confabulation, influence the production of false memories. Extended processing has been shown to make atypical items better remembered than typical items (see, e.g., Lampinen et al., 2001). Perhaps extended processing increases the likelihood that the atypical items are encoded into an ancillary episodic trace. Individuals who score high on measures of dissociation have been found to produce higher rates of false memories (see, e.g., Hekkanen \& McEvoy, 2002; Wilkinson \& Hyman, 1998). Such individuals may have a tendency to encode more information into an ancillary episodic trace. The use of visual imagery has also been shown to increase the number of false memories (see, e.g., Markham \& Hynes, 1993). Imagery, like dissociation and extended processing, could influence how much information is placed into the ancillary trace: As the use of visual imagery increases, the amount of information encoded into the trace increases. In contrast, the effect of forced confabulation (Zaragoza et al., 2001) would operate somewhat differently. When participants were requested to consider whether misinformation occurred in a witnessed event, they were being persuaded to encode information into an ancillary episodic trace. The participants knew it was not part of the witnessed event, so they did not encode the information into the event's episodic trace; rather, they placed it into the ancillary trace for consideration and later forgot that they had been requested to do so.

The results of the present study suggest that two effects occur when an eyewitness procedure is used. The consistency effect is the result of spreading activation in an associative network that makes available implicit associative items. Any test item that is consistent with the schema of the event and is part of the set of activated items will be identified as having occurred in the witnessed event. The suggestion effect shows that the semantic relation between the suggestion and the witnessed event is not always important. Information that is atypical of the witnessed event can be incorporated into recollections of the event as easily as information that is typical. To account for the suggestion effect in the present experiment and other effects that have been observed in false memory research, we propose the inclusion of an ancillary episodic trace in an associative network that is linked to the episodic trace of the witnessed event rather than to the generic schema of the event.

\section{REFERENCES}

Anderson, J. R. (1976). Language, memory, and thought. Hillsdale, NJ: Erlbaum.

Anderson, J. R. (1985). Cognitive psychology and its implications. New York: Freeman.

BeLLI, R. F. (1988). Color blend retrievals: Compromise memories or deliberate compromise responses? Memory \& Cognition, 16, 314-326.

Bower, G. H., Black, J. B., \& Turner, T. J. (1979). Scripts in memory for text. Cognitive Psychology, 11, 177-220.

Brewer, W. F., \& Treyens, J. C. (1981). Role of schemata in memory for places. Cognitive Psychology, 13, 207-230.

DODSON, C. S., \& SCHACTER, D. L. (2002). Aging and strategic retrieval processes: Reducing false memories with a distinctiveness heuristic. Psychology \& Aging, 17, 405-415.

Friedman, A. (1979). Framing pictures: The role of knowledge in automatized encoding and memory for gist. Journal of Experimental Psychology: General, 108, 316-355.

Hekkanen, S. T., \& McEvoy, C. (2002). False memories and sourcemonitoring problems: Criterion differences. Applied Cognitive Psychology, 16, 73-85.

Higham, P. A. (1998). Believing details known to have been suggested. British Journal of Psychology, 89, 265-283.

IsRAel, L., \& Schacter, D. L. (1997). Pictorial encoding reduces false recognition of semantic associates. Psychonomic Bulletin \& Review, 4, 577-581. 
Lampinen, J. M., Copeland, S. M., \& Neuschatz, J. S. (2001). Recollections of things schematic: Room schemas revisited. Journal of Experimental Psychology: Learning, Memory, \& Cognition, 27, 1211-1222.

Lampinen, J. M., \& Schwartz, R. M. (2000). The impersistence of false memory persistence. Memory, 8, 393-400.

LINDSAY, D. S. (1994). Memory source monitoring and eyewitness testimony. In D. F. Ross, J. D. Read, \& M. P. Toglia (Eds.), Adult eyewitness testimony: Current trends and developments (pp. 27-55). New York: Cambridge University Press.

Markham, R., \& Hynes, L. (1993). The effect of vividness of imagery on reality monitoring. Journal of Mental Imagery, 17, 159-170.

McCloskey, M., \& Zaragoza, M. (1985). Misleading postevent information and memory for events: Arguments and evidence against memory impairment hypotheses. Journal of Experimental Psychology: General, 114, 1-16.

McEvoy, C. L., Nelson, D. L., \& Komatsu, T. (1999). What is the connection between true and false memories? The differential roles of interitem associations in recall and recognition. Journal of Experimental Psychology: Learning, Memory, \& Cognition, 25, 1177-1194.

Mitchell, K. J., \& Johnson, M. K. (2000). Source monitoring: Attributing mental experiences. In E. Tulving \& F. I. M. Craik (Eds.), The Oxford handbook of memory (pp. 179-195). New York: Oxford University Press.

Roediger, H. L., III, \& McDermott, K. B. (1995). Creating false memories: Remembering words not presented in lists. Journal of Experimental Psychology: Learning, Memory, \& Cognition, 21, 803-814.

Salasoo, A., Shiffrin, R. M., \& Feustel, T. C. (1985). Building permanent memory codes: Codification and repetition effects in word identification. Journal of Experimental Psychology: General, 114, 50-77.
Schacter, D. L., Israel, L., \& Racine, C. A. (1999). Suppressing false recognition in younger and older adults: The distinctiveness heuristic. Journal of Memory \& Language, 40, 1-24.

SCHANK, R. C. (1982). Dynamic memory: A theory of reminding and learning in computers and people. Cambridge: Cambridge University Press.

Smith, V. L., \& Studebaker, C. A. (1996). What do you expect?: The influence of people's prior knowledge of crime categories on factfinding. Law \& Human Behavior, 20, 517-532.

Thapar, A., \& McDermott, K. B. (2001). False recall and false recognition induced by presentation of associated words: Effects of retention interval and level of processing. Memory \& Cognition, 29, 424-432.

Tousignant, J. P., Hall, D., \& Loftus, E. F. (1986). Discrepancy detection and vulnerability to misleading postevent information. Memory \& Cognition, 14, 329-338.

TuCKey, M. R., \& Brewer, N. (2003). The influence of schemas, stimulus ambiguity, and interview schedule on eyewitness memory over time. Journal of Experimental Psychology: Applied, 9, 101-118.

UNDERWOOD, B. J. (1965). False recognition produced by implicit verbal responses. Journal of Experimental Psychology, 70, 122-129.

WiLKINSON, C., \& HyMAN, I. E., JR. (1998). Individual differences related to two types of memory errors: Word lists may not generalize to autobiographical memory. Applied Cognitive Psychology, 12, S29S46.

Zaragoza, M. S., \& Lane, S. M. (1994). Source misattributions and the suggestibility of eyewitness memory. Journal of Experimental Psychology: Learning, Memory, \& Cognition, 20, 934-945.

Zaragoza, M. S., Payment, K. E., Ackil, J. K., Drivdahl, S. B., \& BECK, M. (2001). Forced confabulation and confirmatory feedback increase false memories. Psychological Science, 12, 473-477.

\begin{tabular}{|c|c|c|c|c|c|}
\hline \multicolumn{6}{|c|}{$\begin{array}{c}\text { APPENDIX } \\
\text { Schema-Typical and Schema-Atypical Items and Their } \\
\text { Mean Typicality Ratings (and Standard Deviations) }\end{array}$} \\
\hline \multicolumn{3}{|c|}{ Schema Typical } & \multicolumn{3}{|c|}{ Schema Atypical } \\
\hline Item & $M$ & $S D$ & Item & $M$ & $S D$ \\
\hline Aspirin bottle & 2.23 & 1.09 & Balloons & 4.17 & 0.89 \\
\hline Briefcase & 1.24 & 0.59 & Candle & 3.83 & 0.97 \\
\hline Clock & 1.09 & 0.47 & Crayons & 3.99 & 0.93 \\
\hline Fax machine & 1.16 & 0.56 & Crutches & 4.40 & 0.79 \\
\hline Floppy disks & 1.24 & 0.66 & Dolls & 4.79 & 0.57 \\
\hline Lamp & 1.56 & 1.06 & Fruit basket & 3.75 & 0.95 \\
\hline Nameplate & 1.43 & 0.75 & Liquor bottles & 4.61 & 0.67 \\
\hline Pager & 1.78 & 0.88 & Pillows & 4.56 & 0.74 \\
\hline Pencil sharpener & 1.36 & 0.62 & Recliner & 4.18 & 0.95 \\
\hline Ruler & 1.84 & 0.87 & Television & 3.72 & 1.08 \\
\hline Scissors & 1.44 & 0.74 & Toaster & 3.99 & 1.05 \\
\hline Tissue box & 1.76 & 0.90 & Toy chest & 4.74 & 0.77 \\
\hline Grand mean & 1.51 & 0.34 & Grand mean & 4.23 & 0.39 \\
\hline
\end{tabular}

Note-A rating of $1=$ very likely to be found in an office, and a rating of $5=$ very unlikely to be found in an office.

(Manuscript received July 25, 2002;

revision accepted for publication August 25, 2004.) 